\title{
High-Q Adaptive Matching Network for Remote Powering of UHF RFIDs and Wireless Sensor Systems
}

\author{
Onur Kazanc $^{1}$, Franco Maloberti ${ }^{2}$, Catherine Dehollain ${ }^{1}$ \\ ${ }^{1}$ RFIC Group, Ecole Polytechnique Federale de Lausanne, Lausanne, Switzerland \\ ${ }^{2}$ Integrated Microsystems Laboratory, University of Pavia, Pavia, Italy \\ e-mail: onur.kazanc@epfl.ch
}

\begin{abstract}
Process and temperature independent RF frontend design is important for remotely powered RFID tags and wireless sensor systems. Run-to-run process variations can impact the remote powering performance and therefore read range of aforementioned systems. We introduce analysis and propose a design methodology for high-Q switched capacitor matching network for remote powering. The method is demonstrated with a differential drive rectifier topology with $-14 \mathrm{dBm}$ of input power at $900 \mathrm{MHz}$ UHF band. Simulation results show the improvement on delivered power up to $42 \%$ and extension of the read range of the sensor system up to $19 \%$ with utilization of the proposed work.
\end{abstract}

\section{INTRODUCTION}

Radio Frequency Identification (RFID) systems are widely used to track, count and monitor the status of various products in supply chains. Mass production of RFID tags enable to utilize such systems at very low cost. Similarly, wireless sensor systems can be used to monitor environmental activity, such as temperature, specific gaseous materials, etc., as well as in medical applications for remote patient monitoring. Passive sensor systems can operate without a battery, meaning that they are remotely powered by their reader, or namely base station, for the necessary amount of energy required.

Far-field passive sensor systems in UHF and microwave frequencies allow long distance operation up to a few meters. This makes them favorable for detecting high quantity of tags with respect to magnetic coupling based near-field sensor systems, which can operate up to a few centimeters distance.

Performance of far-field sensor systems is based on their read range, which indicates the distance between the tag and the base station. The read range of a passive far-field wireless sensor system is highly linked to the impedance matching between the antenna and the rectifier of the integrated system, in other words the total amount of received power. Process variations of integrated circuit technology lead to inevitable mismatch factor between the antenna and the rectifier used for remote powering. This in result, impacts the operation range and overall performance of the deployed tags or sensor systems.

In order to avoid read range degradation due to process originated mismatch, adaptive matching schemes for remotely powered sensor systems had been introduced as exemplified in Fig. 1. O'Driscoll et. al [1] demonstrated a tunable matching network for improving the efficiency of the power link with switched-capacitor banks involving a continuous correction algorithm. However, the quantitative analysis on the matching network is not provided in detail. Bakhtiar et al. [2] proposed a matching network with capacitor bank where capacitors are implemented with MOS capacitors. However, the non-linearity of the MOS capacitance is not taken into account where the input voltage swing across the rectifier might not be large enough to facilitate minimum overdrive voltage for linear operation.

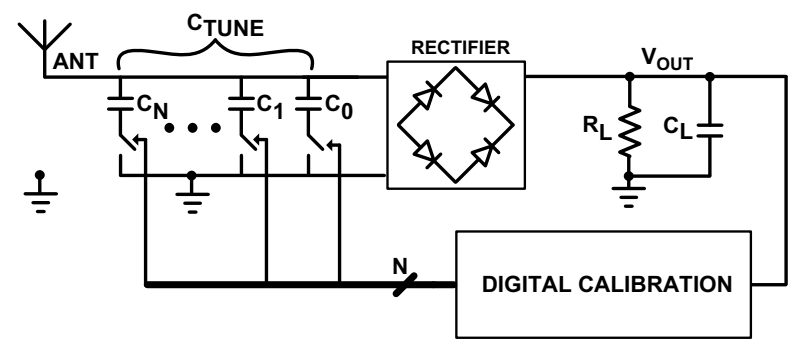

Fig. 1. Impedance tuning scheme with parallel programmable tuning capacitor bank with N-Bits.

In this work, we demonstrate the quantitative influence of switched-capacitor banks on enhancing the power transfer while taking the losses of the matching network into account. We demonstrate a design methodology with a three stage differential rectifier where the power conversion efficiency is high thanks to the differential drive inputs. The design guideline demonstrates its efficacy on the impedance matching while process and temperature variations are in effect.

\section{Impedance MATChing ANd ReAd RANGE}

The read range of a remotely powered wireless sensor system is determined by the minimum input power, namely the sensitivity denoted by $P_{M I N}$. The read range $r$ of a far-field remotely powered system can be calculated by [3],

$$
r=\frac{\lambda}{4 \pi} \sqrt{\frac{P_{E I R P} \cdot G_{R X} \cdot \tau}{P_{M I N}}}
$$


where $P_{E I R P}$ is the effective isotropic radiated power from the reader, $\lambda$ is the wavelength of the electromagnetic wave, $G_{R X}$ is the gain of the receiver antenna and $\tau$ is the power transmission coefficient between the receiving antenna and rectifier. The power transmission coefficient between the antenna and rectifier $\tau$ can be written as [4],

$$
\tau=1-\left|S_{11}\right|^{2}=1-\left|\frac{Z_{C}-Z_{A}^{*}}{Z_{C}+Z_{A}}\right|^{2}
$$

where $\left|S_{11}\right|^{2}$ is power reflection coefficient, $Z_{A}$ and $Z_{C}$ are antenna and chip impedance in the form of $Z=R \pm j X$.

\section{Rectifier InPut Impedance With Process AND TEMPERATURE VARIATION}

The differential rectifier in Fig. 2 is used for RF to DC energy conversion with high efficiency [5]. The rectifier is driven with constant power source which mimics the antenna with a complex impedance of $Z_{A}$ and an available power of $P_{A V}=-14 \mathrm{dBm}$ or $39.8 \mu \mathrm{W}$. A nominal load of $180 \mathrm{k} \Omega$ and a storage capacitor of $1.2 \mathrm{nF}$ are connected to the output of the rectifier. The rectifier is designed and simulated at transistor level in $0.18 \mu \mathrm{m}$ CMOS process. NMOS transistors are sized to $16 / 0.18 \mu \mathrm{m}$ and PMOS transistors are $32 / 0.18 \mu \mathrm{m}$ while MIM capacitor $C_{C}$ is $1 \mathrm{pF}$. The rectifier exhibits $58 \%$ of power conversion efficiency in nominal conditions with almost-ideal impedance matching with the antenna where $S_{11}<-20 \mathrm{~dB}$.

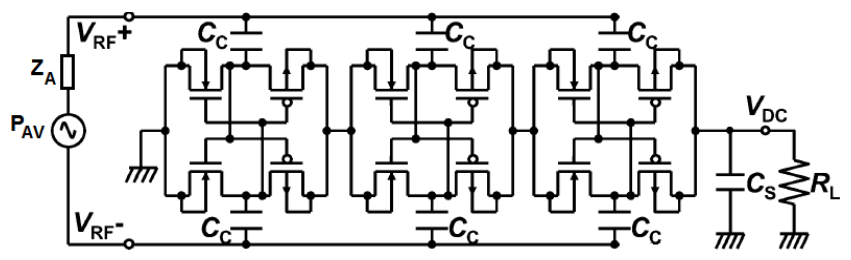

Fig. 2. Three-stage high efficiency differential-drive rectifier[2].

Next we analyze the impact of the process and temperature variations on rectifier input impedance. Based on the simulations, the two extreme process corner cases affecting the input impedance the rectifier is identified as SLOW (mos $=$ ss, mimcaps $=\min , \mathrm{T}=-40^{\circ} \mathrm{C}$ ) and FAST (mos=ff, mimcaps $=\max , \mathrm{T}=80^{\circ} \mathrm{C}$ ) while typical operating condition is denoted as TYP (mos=tt, mimcaps=typ, $\mathrm{T}=27^{\circ} \mathrm{C}$ ). Simulations demonstrate that total variation of $\mp 11.4 \%$ of the input reactance $X_{C}$ of the rectifier from its nominal value is composed of $9.6 \%$ by the process variation and $1.8 \%$ by temperature variation. Fig. 3 depicts the return loss between the rectifier and the antenna, which utilizes inductively coupled feed [6], operating at 900 MHz. It is seen that the resulting reflection coefficient for the two corner cases SLOW and FAST are - $7.6 \mathrm{~dB}$ and -4.9 $\mathrm{dB}$ thus the power absorbed by the rectifier with respect to the nominal condition is decreased by $17 \%$ and $32 \%$ respectively.

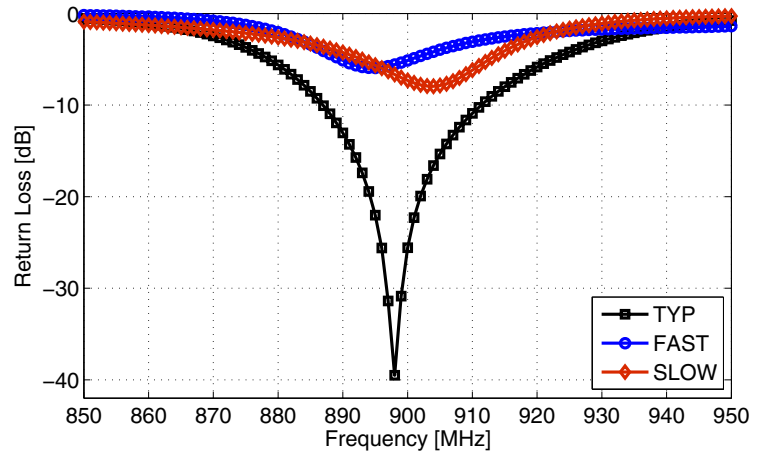

Fig. 3. Return loss between the antenna and the rectifier.

\section{Switched-Capacitor Unit Element For TUNABle MATChING NETWORK}

In order to match the antenna and rectifier along with variations within the defined corners, the tuning scheme introduced in Fig. 1 is utilized with switched-capacitor bank. Note that the differential rectifier topology requires a symmetric tuning element. Therefore, the single-ended unit element shown in Fig. 4 is mirrored with respect to ground for differential operation.

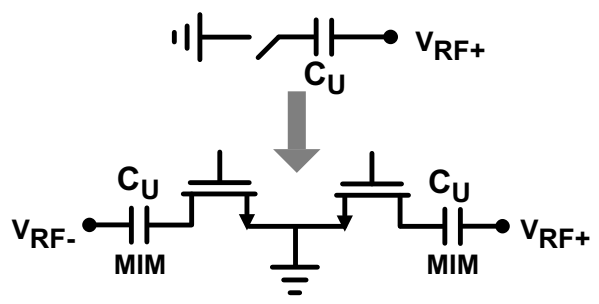

Fig. 4. Switched-capacitor unit element used in the tunable matching network.

The quality factor $(\mathrm{Q})$ and tuning range $(\mathrm{T})$ of tuning element are the most critical parameters on the design of the matching network. Analysis on switched-capacitors is well explained focusing on the trade-off between the tuning range and quality factor [7]. Fig. 5 demonstrates variation of $\mathrm{Q}$ and $\mathrm{T}$ of an example unit element with respect to the transistor width $(\mathrm{W})$ connected in series. As transistor width (W) gets larger, its channel resistance reduces thus improves the quality factor of the unit element. On the other hand, wider transistor has bigger drain junction capacitance that reduces the tuning range of the unit element therefore limits the range of reactive matching.

\section{Tunable Matching Network Design and Simulated Matching Performance}

We have designed a matching network to compensate for the process and temperature variations on the rectifier. In order to choose correct dimensions for the unit element as well as the complete matching network; the corner 


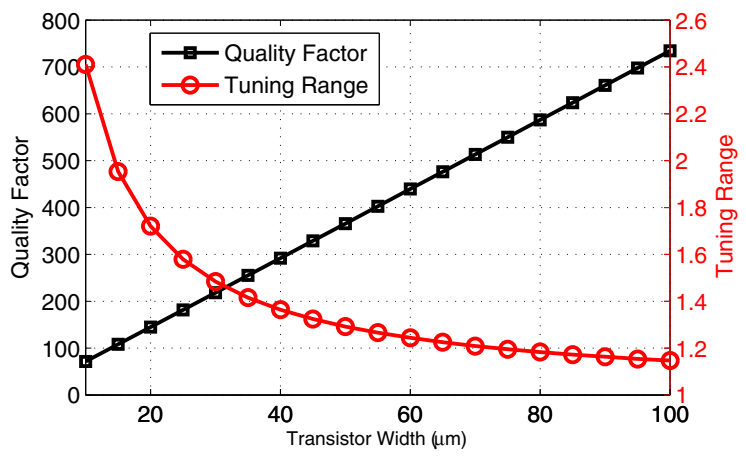

Fig. 5. Dependency of tuning range and quality factor of the capacitor to transistor width.

conditions and certain design issues has to be addressed. Those can be summarized as; a) The matching network should cover full range of variations while considering the variations on the matching network itself. b) The capacitance dimension of the unit element has to be chosen such that it is not dominated by the parasitic capacitance of metal interconnect routes, therefore avoiding reduced tuning range. Those two aforementioned factors determine the capacitance of the unit element and the total number of unit elements in the matching network. Based on the simulation results we have determined the unit capacitance as $C_{U}=14 \mathrm{fF}$, switch transistor with $\mathrm{W}=14 \mu \mathrm{m}$ and $\mathrm{L}=0.18$ $\mu \mathrm{m}$. The capacitor bank of the matching network is composed of 5-bit binary weighted unit elements having total number elements of 31 . Tuning range and quality factor for the chosen capacitor and transistor is simulated at 900 MHz. The resulting tuning range of the unit element is 1.8 and the quality factor is 330 .

The rectifier and the antenna is simulated with the designed matching network placed in between them. Fig. 6 depicts the return loss between the rectifier and the antenna in three cases. While the nominal process TYP performance remains the same at $-22 \mathrm{~dB}$, the return loss for SLOW and FAST corners become $-17.7 \mathrm{~dB}$ and -16.2 $\mathrm{dB}$ respectively. Also, the corresponding absorbed power become $39.3 \mu \mathrm{W}$ and $39.1 \mu \mathrm{W}$ for the two respective corners.

The power efficiency of rectifier with tunable matching method must account the losses in the matching network caused by a finite $\mathrm{Q}$ of the switched-capacitor bank and power required by the state-machine which continuously tracks and tunes the matching network. The power loss in the matching network for nominal corner is $780 \mathrm{nW}$ when half of the unit elements are active. For SLOW corner where all 31 unit elements are active, the total power lost is $1.3 \mu \mathrm{W}$. For FAST corner the power lost reduces to $330 \mathrm{nW}$ when six unit elements out of 31 is active. By estimating a power consumption of $0.5 \mu \mathrm{W}$ for digital control, which is not implemented in this work, the net

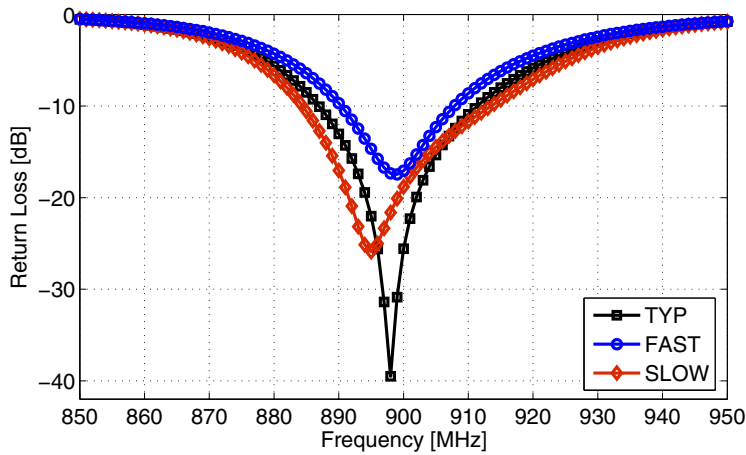

Fig. 6. Return loss between the antenna and the rectifier after implementation of the tunable matching network.

power delivered to rectifier for SLOW corner becomes 37.5 $\mu \mathrm{W}$ for FAST corner $38.3 \mu \mathrm{W}$ increasing the net delivered power to rectifier by $14 \%$ and $42 \%$ respectively. This in result, provide an increase in read range by $7 \%$ for SLOW corner and $19 \%$ in FAST corner according to (1) when compared to untuned matching results.

\section{CONCLUSION}

In this work we demonstrated a design methodology for switched-capacitor tunable matching networks against process variations of the integrated circuit technology as well as temperature variations. The net delivered power to rectifier is improved up to $42 \%$ and read range is improved up to $19 \%$. It is shown that the power lost in tuning elements and estimated overhead power consumption for control circuitry justify implementation of such an adaptive matching system for remotely powered applications.

\section{ACKNOWLEDGMENT}

This work is funded by Nano-Tera initiative of Swiss National Funding (SNF) for project entitled "i-IRONIC".

\section{REFERENCES}

[1] S. O'Driscoll, A. Poon, T.H. Meng, "A mm-Sized Implantable Power Receiver with Adaptive Link Compensation", IEEE International Solid-State Circuits Conference, pp.294-295, Feb 2009

[2] A.S. Bakhtiar, M.S Jalali, S. Mirabbasi, "An RF Power Harvesting System with Input-Tuning for Long-Range RFID Tags", IEEE International Symposium on Circuits and Systems (ISCAS), pp.40854088, 2010

[3] K.V.S. Rao, P.V. Nikitin, S.F. Lam, "Antenna Design for UHF RFID Tags: A Review and a Practical Application", IEEE Transactions on Antennas and Propagation, vol.53, no.12, pp.3870-3876, Dec 2005

[4] P.V. Nikitin et. al, "Power Reflection Coefficient Analysis for Complex Impedances in RFID Tag Design", IEEE Transactions on Microwave Theory and Techniques, vol.53, no.9, pp.2721-2725, Sep 2005

[5] K. Kotani, A. Sasaki, T. Ito, "High-Efficiency Differential-Drive CMOS Rectifier for UHF RFIDs", IEEE Journal of Solid-State Circuits, vol.44, no.11, pp.3011-3018, Nov 2009.

[6] H.W Son, C.S. Pyo, "Design of RFID Tag Antennas Using an Inductively Coupled Feed", Electronics Letters, vol.41, no.18, pp.994-996, Sep. 2005

[7] P. Sjoblom, H. Sjoland, "Measured CMOS Switched High-Quality Capacitors in a Reconfigurable Matching Network", IEEE Trans. on Circuits and Systems II: Express Briefs, vol.54, no.10, pp.858-862, Oct 2007. 